\title{
Erratum to: Policies against human trafficking: the role of religion and political institutions
}

\author{
Niklas Potrafke $e^{1,2}$
}

Published online: 16 September 2016

(C) Springer-Verlag Berlin Heidelberg 2016

\section{Erratum to: Econ Gov DOI 10.1007/s10101-016-0181-5}

The author declares that he used the explanatory variables averaged over the period 1999-2010 instead of the period 2000-2010. Inferences do not change when the explanatory variables averaged over the period 2000-2010 were used.

Please find the corrected Tables 1, 2, 5 and 6 in this erratum.

The online version of the original article can be found under doi:10.1007/s10101-016-0181-5.

$\triangle$ Niklas Potrafke potrafke@ifo.de

1 Department of Economics, University of Munich, Schackstr. 4/I, 80539 Munich, Germany

2 ifo Center for Public Finance and Political Economy, ifo Institute, Poschingerstr. 5, 81679 Munich, Germany 
Table 1 Regression results. Dependent variable: 3P Anti-trafficking Index OLS with robust standard errors

\begin{tabular}{|c|c|c|c|c|}
\hline & (1) & (2) & (3) & (4) \\
\hline & Types of religion & Types of religion & Excluding India & Muslim/Christian \\
\hline Muslims & $\begin{array}{l}-0.0125^{* *} \\
(-2.09)\end{array}$ & $\begin{array}{l}-0.0200^{* * *} \\
(-2.92)\end{array}$ & $\begin{array}{l}-0.0208^{* * *} \\
(-2.98)\end{array}$ & $\begin{array}{l}-0.0195^{\text {*** }} \\
(-2.96)\end{array}$ \\
\hline Buddhists & $\begin{array}{l}0.0165 \\
(1.42)\end{array}$ & $\begin{array}{l}0.00488 \\
(0.42)\end{array}$ & $\begin{array}{l}0.00297 \\
(0.26)\end{array}$ & \\
\hline Hindus & $\begin{array}{l}0.0285^{* *} \\
(2.16)\end{array}$ & $\begin{array}{l}0.0367^{* *} \\
(2.06)\end{array}$ & $\begin{array}{l}0.0255 \\
(1.16)\end{array}$ & \\
\hline Traditional religions & $\begin{array}{l}0.00589 \\
(0.40)\end{array}$ & $\begin{array}{l}0.00294 \\
(0.21)\end{array}$ & $\begin{array}{l}0.00218 \\
(0.16)\end{array}$ & \\
\hline Others & $\begin{array}{l}-0.00278 \\
(-0.13)\end{array}$ & $\begin{array}{l}-0.0269^{*} \\
(-1.70)\end{array}$ & $\begin{array}{l}-0.0294^{*} \\
(-1.85)\end{array}$ & \\
\hline $\begin{array}{l}\text { Others (including } \\
\text { Buddhists etc.) }\end{array}$ & & & & $\begin{array}{l}-0.00260 \\
(-0.30)\end{array}$ \\
\hline $\begin{array}{l}\text { Democracy (Cheibub et } \\
\text { al. 2010) }\end{array}$ & $\begin{array}{l}2.720^{* * *} \\
(4.35)\end{array}$ & $\begin{array}{l}1.066 \\
(1.58)\end{array}$ & $\begin{array}{l}0.901 \\
(1.32)\end{array}$ & $\begin{array}{l}1.102 \\
(1.63)\end{array}$ \\
\hline Muslims*Democracy & $\begin{array}{l}0.00532 \\
(0.60)\end{array}$ & $\begin{array}{l}0.0165 \\
(1.65)\end{array}$ & $\begin{array}{l}0.0169^{*} \\
(1.68)\end{array}$ & $\begin{array}{l}0.0152 \\
(1.53)\end{array}$ \\
\hline Buddhists*Democracy & $\begin{array}{l}-0.0173 \\
(-0.98)\end{array}$ & $\begin{array}{l}-0.0119 \\
(-0.55)\end{array}$ & $\begin{array}{l}-0.0138 \\
(-0.63)\end{array}$ & \\
\hline Hindus*Democracy & $\begin{array}{l}-0.0448^{* *} \\
(-2.24)\end{array}$ & $\begin{array}{l}-0.0471 \\
(-1.64)\end{array}$ & $\begin{array}{l}-0.00689 \\
(-0.21)\end{array}$ & \\
\hline $\begin{array}{l}\text { Traditional religions } \\
\text { *Democracy }\end{array}$ & $\begin{array}{l}-0.0468^{* *} \\
(-2.49)\end{array}$ & $\begin{array}{l}-0.0100 \\
(-0.61)\end{array}$ & $\begin{array}{l}-0.00669 \\
(-0.41)\end{array}$ & \\
\hline Other*Democracy & $\begin{array}{l}0.0162 \\
(0.61)\end{array}$ & $\begin{array}{l}0.0236 \\
(1.38)\end{array}$ & $\begin{array}{l}0.0262 \\
(1.54)\end{array}$ & \\
\hline $\begin{array}{l}\text { Other (including } \\
\text { Buddhism etc.) } \\
\text { *Democracy }\end{array}$ & & & & $\begin{array}{l}-0.00148 \\
(-0.14)\end{array}$ \\
\hline Asia & & $\begin{array}{l}0.396 \\
(0.74)\end{array}$ & $\begin{array}{l}0.533 \\
(1.02)\end{array}$ & $\begin{array}{l}0.577 \\
(1.24)\end{array}$ \\
\hline Europe & & $\begin{array}{l}0.725 \\
(1.01)\end{array}$ & $\begin{array}{l}0.884 \\
(1.27)\end{array}$ & $\begin{array}{l}1.092 \\
(1.64)\end{array}$ \\
\hline America & & $\begin{array}{l}-0.363 \\
(-0.58)\end{array}$ & $\begin{array}{l}-0.265 \\
(-0.43)\end{array}$ & $\begin{array}{l}-0.238 \\
(-0.38)\end{array}$ \\
\hline
\end{tabular}


Table 1 continued

\begin{tabular}{|c|c|c|c|c|}
\hline & (1) & (2) & (3) & (4) \\
\hline & Types of religion & Types of religion & Excluding India & Muslim/Christian \\
\hline Oceania & & $\begin{array}{l}-1.070 \\
(-1.26)\end{array}$ & $\begin{array}{l}-0.879 \\
(-1.03)\end{array}$ & $\begin{array}{l}-0.906 \\
(-1.14)\end{array}$ \\
\hline Legal origin (french) & & $\begin{array}{l}0.314 \\
(1.12)\end{array}$ & $\begin{array}{l}0.265 \\
(0.96)\end{array}$ & $\begin{array}{l}0.225 \\
(0.81)\end{array}$ \\
\hline Legal origin (socialist) & & $\begin{array}{l}1.133^{* *} \\
(2.09)\end{array}$ & $\begin{array}{l}1.064^{* *} \\
(2.02)\end{array}$ & $\begin{array}{l}0.734 \\
(1.40)\end{array}$ \\
\hline Legal origin (german) & & $\begin{array}{l}0.0737 \\
(0.09)\end{array}$ & $\begin{array}{l}0.0725 \\
(0.09)\end{array}$ & $\begin{array}{l}0.0265 \\
(0.03)\end{array}$ \\
\hline $\begin{array}{l}\text { Legal origin } \\
\text { (scandinavian) }\end{array}$ & & $\begin{array}{l}0.117 \\
(0.17)\end{array}$ & $\begin{array}{l}0.125 \\
(0.18)\end{array}$ & $\begin{array}{l}-0.0798 \\
(-0.11)\end{array}$ \\
\hline log per capita GDP & & $\begin{array}{l}-0.0345 \\
(-0.16)\end{array}$ & $\begin{array}{l}-0.0465 \\
(-0.22)\end{array}$ & $\begin{array}{l}-0.144 \\
(-0.72)\end{array}$ \\
\hline $\log$ Population & & $\begin{array}{l}0.494^{* * *} \\
(5.56)\end{array}$ & $\begin{array}{l}0.530^{* * * *} \\
(5.84)\end{array}$ & $\begin{array}{l}0.445^{* * *} \\
(5.20)\end{array}$ \\
\hline OECD & & $\begin{array}{l}0.687 \\
(1.30)\end{array}$ & $\begin{array}{l}0.635 \\
(1.21)\end{array}$ & $\begin{array}{l}0.847 \\
(1.65)\end{array}$ \\
\hline $\begin{array}{l}\text { Women's Economic } \\
\text { Rights }\end{array}$ & & $\begin{array}{l}0.356 \\
(1.01)\end{array}$ & $\begin{array}{l}0.385 \\
(1.11)\end{array}$ & $\begin{array}{l}0.456 \\
(1.35)\end{array}$ \\
\hline Absence of corruption & & $\begin{array}{l}0.368^{* * *} \\
(2.86)\end{array}$ & $\begin{array}{l}0.370^{* * *} \\
(2.88)\end{array}$ & $\begin{array}{l}0.332^{* *} \\
(2.59)\end{array}$ \\
\hline Oil production value & & $\begin{array}{l}-0.0230 \\
(-0.47)\end{array}$ & $\begin{array}{l}-0.0291 \\
(-0.60)\end{array}$ & $\begin{array}{l}-0.0146 \\
(-0.31)\end{array}$ \\
\hline Constant & $\begin{array}{l}7.755^{* * *} \\
(15.27)\end{array}$ & $\begin{array}{l}2.392 \\
(1.24)\end{array}$ & $\begin{array}{l}2.207 \\
(1.15)\end{array}$ & $\begin{array}{l}3.635^{* *} \\
(2.04)\end{array}$ \\
\hline Observations & 175 & 168 & 167 & 168 \\
\hline R-squared & 0.406 & 0.735 & 0.741 & 0.719 \\
\hline
\end{tabular}

Religious reference: Christianity

Absolute value of $\mathrm{t}$ statistics in brackets; * significant at $10 \%$; ** significant at $5 \%$; *** significant at $1 \%$ 
Table 2 Regression results

\begin{tabular}{|c|c|c|c|c|}
\hline & (1) & (2) & (3) & (4) \\
\hline & Religiosity incl. & Protestants & Catholics & POLITY IV \\
\hline Muslims & $\begin{array}{l}-0.0148^{*} \\
(-1.94)\end{array}$ & $\begin{array}{l}-0.0178^{* *} \\
(-2.14)\end{array}$ & $\begin{array}{l}-0.0184^{*} \\
(-1.78)\end{array}$ & $\begin{array}{l}-0.0136^{* *} \\
(-2.30)\end{array}$ \\
\hline Protestants & & $\begin{array}{l}0.00232 \\
(0.14)\end{array}$ & & \\
\hline Catholics & & & $\begin{array}{l}0.00179 \\
(0.13)\end{array}$ & \\
\hline Others & $\begin{array}{l}0.000995 \\
(0.11)\end{array}$ & $\begin{array}{l}-0.00162 \\
(-0.16)\end{array}$ & $\begin{array}{l}-0.00269 \\
(-0.23)\end{array}$ & $\begin{array}{l}-0.00453 \\
(-0.65)\end{array}$ \\
\hline $\begin{array}{l}\text { Democracy (Cheibub et } \\
\text { al. 2010) }\end{array}$ & $\begin{array}{l}1.568^{* *} \\
(2.10)\end{array}$ & $\begin{array}{l}1.599^{*} \\
(1.96)\end{array}$ & $\begin{array}{l}0.377 \\
(0.44)\end{array}$ & \\
\hline Democracy (POLITY IV) & & & & $\begin{array}{l}0.0971 \\
(1.30)\end{array}$ \\
\hline $\begin{array}{l}\text { Muslims*Democracy } \\
\text { (Cheibub) }\end{array}$ & $\begin{array}{l}0.00754 \\
(0.71)\end{array}$ & $\begin{array}{l}0.0105 \\
(0.96)\end{array}$ & $\begin{array}{l}0.0240^{* *} \\
(2.16)\end{array}$ & \\
\hline $\begin{array}{l}\text { Protestants*Democracy } \\
\text { (Cheibub) }\end{array}$ & & $\begin{array}{l}-0.0223 \\
(-1.22)\end{array}$ & & \\
\hline $\begin{array}{l}\text { Catholics*Democracy } \\
\text { (Cheibub) }\end{array}$ & & & $\begin{array}{l}0.0124 \\
(0.86)\end{array}$ & \\
\hline $\begin{array}{l}\text { Others*Democracy } \\
\text { (Cheibub) }\end{array}$ & $\begin{array}{l}-0.00527 \\
(-0.46)\end{array}$ & $\begin{array}{l}-0.00438 \\
(-0.37)\end{array}$ & $\begin{array}{l}0.00750 \\
(0.58)\end{array}$ & \\
\hline $\begin{array}{l}\text { Muslims*Democracy } \\
\text { (POLITY IV) }\end{array}$ & & & & $\begin{array}{l}0.000631 \\
(0.69)\end{array}$ \\
\hline $\begin{array}{l}\text { Others*Democracy } \\
\text { (POLITY IV) }\end{array}$ & & & & $\begin{array}{l}-0.000188 \\
(-0.19)\end{array}$ \\
\hline Asia & $\begin{array}{l}0.729 \\
(1.59)\end{array}$ & $\begin{array}{l}0.483 \\
(1.02)\end{array}$ & $\begin{array}{l}0.532 \\
(1.15)\end{array}$ & $\begin{array}{c}0.968^{*} \\
(1.80)\end{array}$ \\
\hline Europe & $\begin{array}{l}1.543^{* *} \\
(2.20)\end{array}$ & $\begin{array}{l}0.992 \\
(1.44)\end{array}$ & $\begin{array}{l}1.078 \\
(1.57)\end{array}$ & $\begin{array}{l}1.167 \\
(1.57)\end{array}$ \\
\hline America & $\begin{array}{l}0.213 \\
(0.33)\end{array}$ & $\begin{array}{l}-0.236 \\
(-0.38)\end{array}$ & $\begin{array}{l}-0.457 \\
(-0.69)\end{array}$ & $\begin{array}{l}0.0846 \\
(0.12)\end{array}$ \\
\hline
\end{tabular}


Table 2 continued

\begin{tabular}{|c|c|c|c|c|}
\hline & (1) & (2) & (3) & (4) \\
\hline & Religiosity incl. & Protestants & Catholics & POLITY IV \\
\hline \multirow[t]{2}{*}{ Oceania } & 1.570 & -0.567 & -0.818 & -1.003 \\
\hline & $(1.58)$ & $(-0.80)$ & $(-0.98)$ & $(-1.07)$ \\
\hline \multirow[t]{2}{*}{ Legal origin (french) } & 0.0136 & 0.0456 & 0.00489 & 0.137 \\
\hline & $(0.05)$ & $(0.15)$ & $(0.02)$ & $(0.41)$ \\
\hline \multirow[t]{2}{*}{ Legal origin (socialist) } & 0.880 & 0.755 & 0.795 & 0.635 \\
\hline & $(1.53)$ & $(1.48)$ & $(1.53)$ & $(1.18)$ \\
\hline \multirow[t]{2}{*}{ Legal origin (german) } & 0.340 & 0.185 & 0.0838 & 0.0631 \\
\hline & $(0.44)$ & $(0.21)$ & $(0.10)$ & $(0.08)$ \\
\hline \multirow{2}{*}{$\begin{array}{l}\text { Legal origin } \\
\text { (scandinavian) }\end{array}$} & 0.0494 & 1.071 & 0.599 & -0.159 \\
\hline & $(0.06)$ & $(1.28)$ & $(0.80)$ & $(-0.22)$ \\
\hline \multirow[t]{2}{*}{ log per capita GDP } & -0.307 & -0.256 & -0.200 & -0.268 \\
\hline & $(-1.43)$ & $(-1.24)$ & $(-1.01)$ & $(-1.09)$ \\
\hline \multirow[t]{2}{*}{ log Population } & $0.350^{* * *}$ & $0.421^{* * *}$ & $0.434^{* * *}$ & $0.336^{* * *}$ \\
\hline & $(3.67)$ & $(5.01)$ & $(4.90)$ & $(3.26)$ \\
\hline \multirow[t]{2}{*}{ OECD } & 0.816 & $0.883^{*}$ & 0.699 & 0.846 \\
\hline & $(1.60)$ & $(1.71)$ & $(1.33)$ & $(1.57)$ \\
\hline \multirow{2}{*}{$\begin{array}{l}\text { Women's Economic } \\
\text { Rights }\end{array}$} & 0.351 & 0.417 & 0.459 & $0.631^{*}$ \\
\hline & $(0.97)$ & $(1.20)$ & $(1.39)$ & $(1.69)$ \\
\hline \multirow[t]{2}{*}{ Absence of corruption } & $0.424^{* * *}$ & $0.413^{* * *}$ & $0.362^{* * *}$ & $0.368^{* * *}$ \\
\hline & $(2.97)$ & $(3.15)$ & $(2.93)$ & $(2.64)$ \\
\hline \multirow[t]{2}{*}{ Oil production value } & -0.00396 & 0.0162 & 0.00152 & 0.0223 \\
\hline & $(-0.07)$ & $(0.33)$ & $(0.03)$ & $(0.39)$ \\
\hline \multirow[t]{2}{*}{ Religiosity } & 1.427 & & & \\
\hline & $(1.51)$ & & & \\
\hline \multirow[t]{2}{*}{ Constant } & $4.118^{*}$ & $4.444^{* *}$ & $4.086^{* *}$ & $5.456^{* * *}$ \\
\hline & $(1.89)$ & $(2.44)$ & $(2.06)$ & $(2.61)$ \\
\hline Observations & 148 & 168 & 168 & 155 \\
\hline R-squared & 0.723 & 0.728 & 0.728 & 0.698 \\
\hline
\end{tabular}

Dependent variable: 3P Anti-trafficking Index OLS with robust standard errors

Absolute value of $\mathrm{t}$ statistics in brackets; * significant at $10 \%$; ** significant at $5 \%$; *** significant at $1 \%$ 
Table 5 List of countries included

\begin{tabular}{|c|c|c|c|c|c|c|c|c|c|}
\hline Country & $3 \mathrm{P}$ & $\begin{array}{l}\text { Christi- } \\
\text { anity }\end{array}$ & Islam & $\begin{array}{l}\text { Demo- } \\
\text { cracy }\end{array}$ & Country & $3 \mathrm{P}$ & $\begin{array}{l}\text { Christi- } \\
\text { anity }\end{array}$ & Islam & $\begin{array}{l}\text { Demo- } \\
\text { cracy }\end{array}$ \\
\hline Afghanistan & 6.7 & 0 & 89 & 0 & Colombia & 11.9 & 93 & 0 & 1 \\
\hline Albania & 11.5 & 33 & 65 & 1 & Comoros & 6 & 0.6 & 99 & 0.56 \\
\hline Algeria & 4.6 & 0.4 & 99 & 0 & Congo & 7 & 78 & 1 & 0 \\
\hline Angola & 7.4 & 90 & 0 & 0 & Costa Rica & 9.5 & 88 & 0 & 1 \\
\hline $\begin{array}{l}\text { Antigua and } \\
\text { Barbuda }\end{array}$ & 8.7 & 96 & 0 & 1 & Cote d'Ivoire & 7.8 & 20 & 20 & 0 \\
\hline Argentina & 9.9 & 95 & 0 & 1 & Croatia & 12.2 & 89 & 1 & 1 \\
\hline Armenia & 10.1 & 97 & 3 & 1 & Cuba & 4.2 & 36 & 0 & 0 \\
\hline Australia & 14.1 & 74 & 0 & 1 & Cyprus & 10.3 & 78 & 19 & 1 \\
\hline Austria & 13.7 & 88 & 2 & 1 & Czech Republic & 12.5 & 48 & 0 & 1 \\
\hline Azerbaijan & 8.9 & 11 & 80 & 0 & $\begin{array}{l}\text { Democratic Peo- } \\
\text { ple's Republic of } \\
\text { Korea }\end{array}$ & 3 & 0.9 & 0 & 0 \\
\hline Bahamas & 6.2 & 83 & 0 & 1 & $\begin{array}{l}\text { Democratic Repub- } \\
\text { lic of the Congo }\end{array}$ & 7.3 & 94 & 1 & 0 \\
\hline Bahrain & 6.8 & 7 & 90 & 0 & Denmark & 13.7 & 89 & 1 & 1 \\
\hline Bangladesh & 11.1 & 0 & 87 & 0.78 & Djibouti & 6.1 & 5 & 94 & 0 \\
\hline Barbados & 5.8 & 59 & 0 & 1 & $\begin{array}{r}\text { Dominican } \\
\text { Republic }\end{array}$ & 9.1 & 92 & 0 & 1 \\
\hline Belarus & 9.5 & 68 & 0 & 0 & Ecuador & 9.9 & 92 & 0 & 0.78 \\
\hline Belgium & 14.5 & 79 & 3 & 1 & Egypt & 5.9 & 10 & 88 & 0 \\
\hline Belize & 8.9 & 89 & 0 & 1 & El Salvador & 9.9 & 92 & 0 & 1 \\
\hline Benin & 9.5 & 23 & 13 & 1 & $\begin{array}{c}\text { Equatorial } \\
\text { Guinea }\end{array}$ & 7.7 & 85 & 0.5 & 0 \\
\hline Bolivia & 8.7 & 93 & 0 & 1 & Eritrea & 4 & 20 & 80 & 0 \\
\hline $\begin{array}{l}\text { Bosnia and } \\
\text { Herzegovina }\end{array}$ & 11 & 52 & 43 & 0 & Estonia & 8.9 & 100 & 0 & 1 \\
\hline Botswana & 6.8 & 48 & 0 & 0 & Ethiopia & 9.7 & 53 & 32 & 0 \\
\hline Brazil & 11 & 78 & 0 & 1 & Fiji & 7.2 & 52 & 8 & 0 \\
\hline $\begin{array}{l}\text { Brunei } \\
\text { Darussalam }\end{array}$ & 5.7 & 10 & 63 & 0 & Finland & 11.9 & 88 & 0 & 1 \\
\hline Bulgaria & 11 & 78 & 13 & 1 & France & 12.7 & 82 & 3 & 1 \\
\hline Burkina Faso & 9.5 & 12 & 42 & 0 & Gabon & 10.2 & 96 & 0.8 & 0 \\
\hline Burundi & 7.7 & 79 & 1 & 0.44 & Gambia & 7.6 & 4 & 94 & 0 \\
\hline Cambodia & 9.2 & 0 & 2 & 0 & Georgia & 10.2 & 83 & 11 & 0.56 \\
\hline Cameroon & 7.6 & 52 & 22 & 0 & Germany & 14.6 & 61 & 2 & 1 \\
\hline Canada & 12.6 & 87 & 0.400 & 1 & Ghana & 9.8 & 62 & 16 & 1 \\
\hline $\begin{array}{l}\text { Central African } \\
\text { Republic }\end{array}$ & 6.5 & 69 & 8 & 0.33 & Greece & 9.8 & 98 & 2 & 1 \\
\hline Chad & 6.9 & 33 & 43 & 0 & Guatemala & 10.1 & 98 & 0 & 1 \\
\hline Chile & 11.1 & 87 & 0 & 1 & Guinea & 8.5 & 2 & 80 & 0 \\
\hline China & 10.3 & 0.2 & 2 & 0 & $\begin{array}{l}\text { Guinea } \\
\text { Bissau }\end{array}$ & 7.7 & 6 & 34 & 0.89 \\
\hline
\end{tabular}


Table 5 continued

\begin{tabular}{|c|c|c|c|c|c|c|c|c|c|}
\hline Country & $3 \mathrm{P}$ & $\begin{array}{l}\text { Christi- } \\
\text { anity }\end{array}$ & Islam & $\begin{array}{l}\text { Demo- } \\
\text { cracy }\end{array}$ & Country & $3 \mathrm{P}$ & $\begin{array}{l}\text { Christi- } \\
\text { anity }\end{array}$ & Islam & $\begin{array}{l}\text { Demo- } \\
\text { cracy }\end{array}$ \\
\hline Guyana & 9 & 41 & 0 & 0 & Malta & 9.4 & 97 & 0 & 1 \\
\hline Haiti & 6 & 94 & 0 & 0 & Mauritania & 7.3 & 0.4 & 99 & 0.11 \\
\hline Honduras & 8.9 & 98 & 0 & 1 & Mauritius & 10.9 & 30 & 13 & 1 \\
\hline Hungary & 10.6 & 82 & 0 & 1 & Mexico & 9 & 96 & 0 & 1 \\
\hline Iceland & 10.3 & 97 & 0 & 1 & Mongolia & 9 & 0 & 1 & 1 \\
\hline India & 9.5 & 2 & 11 & 1 & Montenegro & 9.5 & 70 & 19 & 0 \\
\hline Indonesia & 9 & 9 & 81 & 1 & Morocco & 9 & 1 & 99 & 0 \\
\hline Iran & 6.3 & 0.7 & 98 & 0 & Mozambique & 8.4 & 31 & 13 & 0 \\
\hline Iraq & 4.1 & 4 & 94 & 0 & Myanmar & 7 & 5 & 4 & 0 \\
\hline Ireland & 11.7 & 96 & 0 & 1 & Namibia & 7.5 & 82 & 0 & 0 \\
\hline Israel & 10.4 & 2 & 10 & 1 & Nepal & 10.3 & 0.2 & 3 & 0.33 \\
\hline Italy & 13.8 & 82 & 0 & 1 & Netherlands & 14.2 & 62 & 3 & 1 \\
\hline Jamaica & 9.3 & 58 & 0 & 1 & New Zealand & 12.1 & 63 & 0 & 1 \\
\hline Japan & 9.5 & 2 & 0 & 1 & Nicaragua & 9.1 & 92 & 0 & 1 \\
\hline Jordan & 7.3 & 5 & 92 & 0 & Nigeria & 11 & 49 & 45 & 1 \\
\hline Kazakhstan & 9.7 & 17 & 47 & 0 & Niger & 8.6 & 0 & 90 & 1 \\
\hline Kenya & 9 & 73 & 6 & 1 & Norway & 14.3 & 92 & 0 & 1 \\
\hline Kiribati & 4.75 & 95 & 0 & 1 & Oman & 7.9 & 0 & 87 & 0 \\
\hline Kuwait & 6.3 & 0 & 85 & 0 & Pakistan & 9.3 & 2 & 97 & 0.11 \\
\hline Kyrgyzstan & 8.9 & 20 & 70 & 0.44 & Panama & 10.1 & 89 & 5 & 1 \\
\hline $\begin{array}{c}\text { Lao People's } \\
\text { Democratic } \\
\text { Republic }\end{array}$ & 9.2 & 2 & 1 & 0 & $\begin{array}{l}\text { Papua New } \\
\text { Guinea }\end{array}$ & 6.2 & 91 & 0 & 1 \\
\hline Latvia & 10.7 & 65 & 0 & 1 & Paraguay & 9.8 & 98 & 0 & 1 \\
\hline Lebanon & 6 & 15 & 50 & 0 & Peru & 9.5 & 98 & 0 & 0.89 \\
\hline Lesotho & 7 & 93 & 0 & 0 & Philippines & 11.5 & 88 & 4 & 1 \\
\hline Liberia & 7.4 & 67 & 14 & 0.33 & Poland & 12.5 & 90 & 0 & 1 \\
\hline Libya & 4.6 & 0 & 97 & 0 & Portugal & 12.6 & 94 & 0.1 & 1 \\
\hline Lithuania & 12.4 & 95 & 0 & 1 & Qatar & 6.1 & 6 & 92 & 0 \\
\hline Luxembourg & 12.4 & 93 & 0 & 1 & $\begin{array}{l}\text { Republic of } \\
\text { Korea }\end{array}$ & 13.3 & 33 & 0 & 1 \\
\hline Macedonia & 11.6 & 64 & 26 & 1 & $\begin{array}{c}\text { Republic of } \\
\text { Moldova }\end{array}$ & 9.6 & 97 & 0 & 1 \\
\hline Madagascar & 8.8 & 50 & 3 & 1 & Romania & 11.4 & 90 & 0.2 & 1 \\
\hline Malawi & 10.7 & 64 & 16 & 1 & $\begin{array}{l}\text { Russian } \\
\text { Federa- } \\
\text { tion }\end{array}$ & 9.1 & 84 & 5 & 0 \\
\hline Malaysia & 7.9 & 6 & 53 & 0 & Rwanda & 9.6 & 74 & 9 & 0 \\
\hline Maldives & 4.7 & 0 & 100 & 0.11 & $\begin{array}{l}\text { Saint Vincent } \\
\text { and the } \\
\text { Grenadines }\end{array}$ & 6.3 & 90 & 0 & 1 \\
\hline Mali & 8.3 & 1 & 89 & 1 & Saint Lucia & 8 & 95 & 0 & 1 \\
\hline
\end{tabular}


Table 5 continued

\begin{tabular}{|c|c|c|c|c|c|c|c|c|c|}
\hline Country & $3 \mathrm{P}$ & $\begin{array}{l}\text { Christi- } \\
\text { anity }\end{array}$ & Islam & $\begin{array}{l}\text { Demo- } \\
\text { cracy }\end{array}$ & Country & $3 \mathrm{P}$ & $\begin{array}{l}\text { Christi- } \\
\text { anity }\end{array}$ & Islam & $\begin{array}{l}\text { Demo- } \\
\text { cracy }\end{array}$ \\
\hline Saudi Arabia & 6.3 & 0.8 & 99 & 0 & Thailand & 11.8 & 1 & 4 & 0.78 \\
\hline Senegal & 9.6 & 5 & 91 & 1 & Togo & 8.1 & 35 & 12 & 0 \\
\hline Serbia & 9.1 & 70 & 19 & 1 & Tonga & 6 & 93 & 0 & 0 \\
\hline Seychelles & 6 & 97 & 0 & 0 & $\begin{array}{l}\text { Trinidad and } \\
\text { Tobago }\end{array}$ & 7 & 55 & 6 & 1 \\
\hline Sierra Leone & 7.6 & 8 & 39 & 1 & Tunisia & 5.9 & 0.3 & 99 & 0 \\
\hline Singapore & 8.8 & 16 & 16 & 0 & Turkey & 10.2 & 0.3 & 99 & 1 \\
\hline Slovakia & 10.6 & 80 & 0 & 1 & Turkmenistan & 4.2 & 11 & 87 & 0 \\
\hline Slovenia & 11.9 & 96 & 1 & 1 & Uganda & 8.3 & 76 & 7 & 0 \\
\hline $\begin{array}{r}\text { Solomon } \\
\text { Islands }\end{array}$ & 4 & 93 & 0 & 1 & Ukraine & 10.3 & 90 & 8 & 1 \\
\hline Somalia & 3.2 & 0.1 & 100 & 0 & $\begin{array}{l}\text { United Arab } \\
\text { Emirates }\end{array}$ & 8.7 & 4 & 95 & 0 \\
\hline South Africa & 7.4 & 68 & 1 & 0 & $\begin{array}{l}\text { United } \\
\text { Kingdom }\end{array}$ & 13.1 & 87 & 1 & 1 \\
\hline Spain & 13.6 & 96 & 0 & 1 & $\begin{array}{l}\text { United Republic } \\
\text { of Tanzania }\end{array}$ & 8.8 & 34 & 33 & 0 \\
\hline Sri Lanka & 9.5 & 8 & 8 & 1 & United States & 15 & 62 & 2 & 1 \\
\hline Sudan & 4.5 & 8 & 73 & 0 & Uruguay & 9.9 & 62 & 0 & 1 \\
\hline Suriname & 8.4 & 41 & 20 & 1 & Uzbekistan & 9.6 & 9 & 88 & 0 \\
\hline Swaziland & 6.4 & 77 & 0 & 0 & Venezuela & 7.3 & 91 & 0 & 1 \\
\hline Sweden & 13.7 & 80 & 0 & 1 & Viet Nam & 11.1 & 7 & 0 & 0 \\
\hline Switzerland & 12.4 & 92 & 2 & 1 & Yemen & 7.1 & 0 & 100 & 0 \\
\hline Syria & 5.6 & 8 & 90 & 0 & Zambia & 10 & 69 & 0.300 & 0 \\
\hline Taiwan & 10.2 & 7 & 0.5 & 1 & Zimbabwe & 7.3 & 43 & 0 & 0 \\
\hline Tajikistan & 9.3 & 0 & 85 & 0 & Total & 9 & 51.59 & 25.57 & 0.56 \\
\hline
\end{tabular}


Table 6 Data description and sources

\begin{tabular}{|c|c|c|c|c|c|c|}
\hline Variable & Observations & Mean & Std. Dev. & Min & Max & Source \\
\hline $\begin{array}{l}\text { 3P index } \\
\text { (prosecution) }\end{array}$ & 175 & 3.35 & 1.07 & 1 & 5 & Cho et al. (2014) \\
\hline $\begin{array}{l}\text { 3P index } \\
\text { (protection) }\end{array}$ & 175 & 2.64 & 0.89 & 1 & 5 & Cho et al. (2014) \\
\hline $\begin{array}{l}\text { 3P index } \\
\text { (prevention) }\end{array}$ & 175 & 3.05 & 0.83 & 1 & 5 & Cho et al. (2014) \\
\hline 3P index (overall) & 175 & 9.04 & 2.54 & 3 & 15 & Cho et al. (2014) \\
\hline Democracy & 175 & 0.56 & 0.48 & 0 & 1 & $\begin{array}{l}\text { Cheibub et al. } \\
\text { (2010) }\end{array}$ \\
\hline Anglicans share & 175 & 2.31 & 8.11 & 0 & 57 & Parker (1997) \\
\hline Buddhists share & 175 & 3.59 & 14.93 & 0 & 92 & Parker (1997) \\
\hline Christians share & 175 & 51.59 & 38.03 & 0 & 100 & Parker (1997) \\
\hline Hindus share & 175 & 2.36 & 10.84 & 0 & 86 & Parker (1997) \\
\hline Jews share & 175 & 0.58 & 6.35 & 0 & 84 & Parker (1997) \\
\hline Muslims share & 175 & 25.57 & 36.82 & 0 & 100 & Parker (1997) \\
\hline Protestants share & 175 & 13.86 & 23.06 & 0 & 100 & Parker (1997) \\
\hline $\begin{array}{l}\text { Roman Catholics } \\
\text { share }\end{array}$ & 175 & 27.54 & 34.25 & 0 & 97 & Parker (1997) \\
\hline $\begin{array}{l}\text { Traditional } \\
\text { religions share }\end{array}$ & 175 & 5.60 & 12.67 & 0 & 63 & Parker (1997) \\
\hline Christian majority & 175 & 0.59 & 0.49 & 0 & 1 & $\begin{array}{l}\text { CIA World } \\
\text { Factbook (2013) }\end{array}$ \\
\hline Muslim majority & 175 & 0.29 & 0.45 & 0 & 1 & $\begin{array}{l}\text { CIA World } \\
\text { Factbook (2013) }\end{array}$ \\
\hline Hindu majority & 175 & 0.02 & 0.15 & 0 & 1 & $\begin{array}{l}\text { CIA World } \\
\text { Factbook (2013) }\end{array}$ \\
\hline Buddhist majority & 175 & 0.05 & 0.21 & 0 & 1 & $\begin{array}{l}\text { CIA World } \\
\text { Factbook (2013) }\end{array}$ \\
\hline Jewish majority & 175 & 0.01 & 0.08 & 0 & 1 & $\begin{array}{l}\text { CIA World } \\
\text { Factbook (2013) }\end{array}$ \\
\hline $\begin{array}{c}\text { Indigenous } \\
\text { majority }\end{array}$ & 175 & 0.05 & 0.21 & 0 & 1 & $\begin{array}{l}\text { CIA World } \\
\text { Factbook (2013) }\end{array}$ \\
\hline Religiosity & 152 & 0.72 & 0.25 & 0.15 & 1 & $\begin{array}{l}\text { Berggren } \\
\text { \&Bjørnskov } \\
(2013)\end{array}$ \\
\hline British legal origin & 173 & 0.31 & 0.46 & 0 & 1 & $\begin{array}{l}\text { La Porta et al. } \\
\text { (1999) }\end{array}$ \\
\hline $\begin{array}{l}\text { French legal } \\
\text { origin }\end{array}$ & 173 & 0.43 & 0.50 & 0 & 1 & $\begin{array}{l}\text { La Porta et al. } \\
\text { (1999) }\end{array}$ \\
\hline $\begin{array}{l}\text { Socialist legal } \\
\text { origin }\end{array}$ & 173 & 0.20 & 0.40 & 0 & 1 & $\begin{array}{l}\text { La Porta et al. } \\
\text { (1999) }\end{array}$ \\
\hline $\begin{array}{l}\text { German legal } \\
\text { origin }\end{array}$ & 173 & 0.03 & 0.18 & 0 & 1 & $\begin{array}{l}\text { La Porta et al. } \\
\text { (1999) }\end{array}$ \\
\hline $\begin{array}{r}\text { Scandinavian } \\
\text { legal origin }\end{array}$ & 173 & 0.03 & 0.17 & 0 & 1 & $\begin{array}{l}\text { La Porta et al. } \\
\text { (1999) }\end{array}$ \\
\hline
\end{tabular}


Table 6 continued

\begin{tabular}{|c|c|c|c|c|c|c|}
\hline Variable & Observations & Mean & Std. Dev. & Min & Max & Source \\
\hline Africa & 175 & 0.29 & 0.46 & 0 & 1 & own calculation \\
\hline Americas & 175 & 0.18 & 0.39 & 0 & 1 & own calculation \\
\hline Asia & 175 & 0.26 & 0.44 & 0 & 1 & own calculation \\
\hline Europe & 175 & 0.23 & 0.42 & 0 & 1 & own calculation \\
\hline Oceania & 175 & 0.04 & 0.20 & 0 & 1 & own calculation \\
\hline OECD & 175 & 0.17 & 0.38 & 0 & 1 & own calculation \\
\hline Population & 173 & 36846.00 & 132687.15 & 81.20 & 1297335.50 & $\begin{array}{l}\text { PENN World Table } \\
\text { 7.1 -, Summers } \\
\text { \&Heston (1991) }\end{array}$ \\
\hline $\begin{array}{l}\text { Real GDP per } \\
\text { capita }\end{array}$ & 173 & 12014.37 & 14572.09 & 208.40 & 84452.09 & $\begin{array}{l}\text { PENN World Table } \\
7.1 \text { - Summers } \\
\text { \&Heston (1991) }\end{array}$ \\
\hline $\begin{array}{l}\text { Oil production } \\
\text { value per } \\
\text { capita }\end{array}$ & 174 & 700.62 & 2483.07 & 0 & 19269.50 & $\begin{array}{l}\text { EIA (2012), own } \\
\text { calculation }\end{array}$ \\
\hline $\begin{array}{l}\text { Democracy } \\
\text { (type2) }\end{array}$ & 175 & 0.78 & 0.39 & 0 & 1 & $\begin{array}{l}\text { Cheibub et al. } \\
\text { (2010) }\end{array}$ \\
\hline $\begin{array}{l}\text { POLITY IV } \\
\text { index }\end{array}$ & 160 & 3.42 & 6.32 & -10 & 10 & $\begin{array}{l}\text { Marshall \&Jaggers } \\
\quad(2011)\end{array}$ \\
\hline $\begin{array}{l}\text { POLITY IV } \\
\text { executive } \\
\text { constraints }\end{array}$ & 145 & 4.89 & 2.01 & 1 & 7 & $\begin{array}{l}\text { Marshall \&Jaggers } \\
\quad \text { (2011) }\end{array}$ \\
\hline $\begin{array}{l}\text { KOF index of } \\
\text { globalization } \\
\text { (overall) }\end{array}$ & 171 & 55.81 & 16.96 & 21.99 & 92.43 & $\begin{array}{l}\text { Dreher (2006); } \\
\text { Dreher et al. } \\
(2008)\end{array}$ \\
\hline $\begin{array}{l}\text { KOF index of } \\
\text { globalization } \\
\text { (economic) }\end{array}$ & 146 & 60.49 & 16.87 & 26.40 & 96.27 & $\begin{array}{l}\text { Dreher (2006); } \\
\text { Dreher et al. } \\
(2008)\end{array}$ \\
\hline $\begin{array}{l}\text { KOF index of } \\
\text { globalization } \\
\text { (social) }\end{array}$ & 171 & 47.59 & 21.79 & 12.31 & 92.18 & $\begin{array}{l}\text { Dreher (2006); } \\
\text { Dreher et al. } \\
(2008)\end{array}$ \\
\hline $\begin{array}{l}\text { KOF index of } \\
\text { globalization } \\
\text { (political) }\end{array}$ & 174 & 64.57 & 20.34 & 20.69 & 97.30 & $\begin{array}{l}\text { Dreher (2006); } \\
\text { Dreher et al. } \\
(2008)\end{array}$ \\
\hline $\begin{array}{l}\text { Absence of } \\
\text { corruption }\end{array}$ & 172 & 3.97 & 2.09 & 1.33 & 9.52 & $\begin{array}{l}\text { Transparency } \\
\text { International } \\
\text { (2012) }\end{array}$ \\
\hline $\begin{array}{l}\text { Women's } \\
\text { economic } \\
\text { rights }\end{array}$ & 175 & 1.30 & 0.60 & 0 & 3 & $\begin{array}{l}\text { Cingranelli } \\
\text { \&Richards } \\
\text { (2010) }\end{array}$ \\
\hline $\begin{array}{l}\text { Trafficking } \\
\text { (transit) }\end{array}$ & 150 & 1.44 & 1.51 & 0 & 5 & UNODC (2006) \\
\hline $\begin{array}{l}\text { Net migration } \\
\text { flows }\end{array}$ & 171 & -797.58 & 686460.99 & -1917599.62 & 6557193.00 & Worldbank (2011) \\
\hline Migrant stock & 174 & 7.66 & 12.04 & 0.05 & 80.24 & Worldbank (2011) \\
\hline
\end{tabular}

